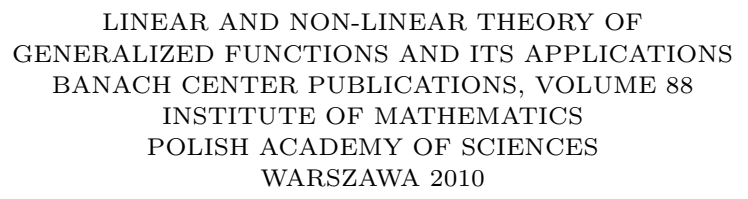

\title{
WEYL PRODUCT ALGEBRAS AND CLASSICAL MODULATION SPACES
}

\author{
ANDERS HOLST \\ Department of Mathematics, Lund University \\ 22100 Lund, Sweden \\ E-mail: anders.holst@math.lth.se \\ JOACHIM TOFT \\ Department of Mathematics and Systems Engineering, Växjö University \\ 35195 Växjö, Sweden \\ E-mail: joachim.toft@vxu.se \\ PATRIK WAHLBERG \\ School of Electrical Engineering and Computer Science, University of Newcastle \\ Callaghan, NSW 2308, Australia \\ E-mail: patrik.wahlberg@newcastle.edu.au
}

\begin{abstract}
We discuss continuity properties of the Weyl product when acting on classical modulation spaces. In particular, we prove that $M^{p, q}$ is an algebra under the Weyl product when $p \in[1, \infty]$ and $1 \leq q \leq \min \left(p, p^{\prime}\right)$.

1. Introduction. The aim of this paper is to investigate algebraic properties of the Weyl product on classical modulation spaces 1 , i. e. unweighted modulation spaces. These investigations go back to [10, where similar properties are done for general modulation spaces (weighted modulation spaces). In fact, in [10] a general result is presented, which contains most of the existing results in this context (cf. [12, 14, 15]), concerning conditions that are necessary and sufficient for modulation spaces to be algebras under this product.
\end{abstract}

2000 Mathematics Subject Classification: Primary 42B35, 35S05; Secondary 47B37.

Key words and phrases: Weyl calculus, pseudo-differential calculus, modulation spaces, Banach algebras.

The paper is in final form and no version of it will be published elsewhere.

${ }^{1}$ The term "classical" appeared after some considerations within the time-frequency community. See also [3]. 
The (classical) modulation spaces $M^{p, q}, p, q \in[1, \infty]$, as introduced by Feichtinger in [2], consist of all tempered distributions whose short-time Fourier transforms (STFT) have finite mixed $L^{p, q}$ norm. It follows that the parameters $p$ and $q$ to some extent quantify the degrees of asymptotic decay and singularity of the distributions in $M^{p, q}$. From the construction of these spaces, it turns out that modulation spaces and Besov spaces in some sense are rather similar, and sharp embeddings between these spaces can be found in [16, 18].

In [14, Sjöstrand introduced the modulation space $M^{\infty, 1}$, which contains non-smooth functions, as a symbol class. He proved that the symbol class $M^{\infty, 1}$ corresponds to an algebra of operators which are bounded on $L^{2}$. Gröchenig and Heil thereafter proved in [7. 6] that such operators are continuous on all modulation spaces $M^{p, q}, p, q \in[1, \infty]$. This extends Sjöstrand's result since $M^{2,2}=L^{2}$. Some generalizations to operators with symbols in a broader class of classical modulation spaces were then obtained in [8, 16], and in [17, 19] some further extensions involving weighted modulation spaces are presented.

Within the theory of pseudo-differential operators, the Weyl quantization seems to be natural to use in many contexts. In fact, such operators can be formulated in a way which is independent of the choice of symplectic coordinates, a property which is important in quantum mechanics. It also seems that the most natural way to pass from classical mechanics to quantum mechanics, is to use Weyl quantization, where the observable $a(x, \xi)$ in classical mechanics corresponds to the Weyl operator $a^{w}(x, D)$ in quantum mechanics (with "good" approximation).

Here we recall that if $a \in \mathscr{S}\left(\mathbf{R}^{2 d}\right)$, then the Weyl quantization $a^{w}(x, D)$ with symbol $a$ is defined by the formula

$$
a^{w}(x, D) f(x)=(2 \pi)^{-d} \iint a((x+y) / 2, \xi) e^{i\langle x-y, \xi\rangle} f(y) d y d \xi
$$

where $f \in \mathscr{S}\left(\mathbf{R}^{d}\right)$. The definition in $(1)$ extends to any $a \in \mathscr{S}^{\prime}\left(\mathbf{R}^{2 d}\right)$, and then $a_{t}(x, D)$ is continuous from $\mathscr{S}\left(\mathbf{R}^{d}\right)$ to $\mathscr{S}^{\prime}\left(\mathbf{R}^{d}\right)$. (See also [1].)

Next assume that $a, b \in \mathscr{S}^{\prime}\left(\mathbf{R}^{2 d}\right)$ are such that the composition $a^{w}(x, D) \circ b^{w}(x, D)$ makes sense as a continuous operator from $\mathscr{S}\left(\mathbf{R}^{d}\right)$ to $\mathscr{S}^{\prime}\left(\mathbf{R}^{d}\right)$. Then by the Schwartz kernel theorem and the Weyl quantization, there is a unique distribution $c \in \mathscr{S}^{\prime}\left(\mathbf{R}^{2 d}\right)$ such that $c^{w}(x, D)=a^{w}(x, D) \circ b^{w}(x, D)$. In this case we define the Weyl product between $a$ and $b$ as $a \# b=c$. Hence, the Weyl product is the multiplication between symbol pairs defined by $(a, b) \mapsto a \# b$, and it follows that it is well-defined if, for example, $a \in \mathscr{S}^{\prime}\left(\mathbf{R}^{2 d}\right)$ and $b \in \mathscr{S}\left(\mathbf{R}^{2 d}\right)$. The Weyl product can also be defined for other pairs of tempered distributions $a, b \in \mathscr{S}^{\prime}\left(\mathbf{R}^{2 d}\right)$.

Especially we are concerned with finding sufficient conditions on $p_{j}, q_{j} \in[1, \infty]$, in order for the map

$$
\mathscr{S}\left(\mathbf{R}^{2 d}\right) \times \mathscr{S}\left(\mathbf{R}^{2 d}\right) \ni(a, b) \mapsto a \# b \in \mathscr{S}\left(\mathbf{R}^{2 d}\right)
$$

to be uniquely extendable to a map from $M^{p_{1}, q_{1}}\left(\mathbf{R}^{2 d}\right) \times M^{p_{2}, q_{2}}\left(\mathbf{R}^{2 d}\right)$ to $M^{p_{0}, q_{0}}\left(\mathbf{R}^{2 d}\right)$, which is continuous in the sense that for some constant $C>0$,

$$
\|a \# b\|_{M^{p_{0}, q_{0}}} \leq C\|a\|_{M^{p_{1}, q_{1}}}\|b\|_{M^{p_{2}, q_{2}}},
$$

when $a \in M^{p_{1}, q_{1}}\left(\mathbf{R}^{2 d}\right)$ and $b \in M^{p_{2}, q_{2}}\left(\mathbf{R}^{2 d}\right)$. 
One of Sjöstrand's results can then be formulated as $M^{\infty, 1}\left(\mathbf{R}^{2 d}\right) \# M^{\infty, 1}\left(\mathbf{R}^{2 d}\right) \subseteq$ $M^{\infty, 1}\left(\mathbf{R}^{2 d}\right)$. Sjöstrand's result was refined in [15], where the inclusion

$$
M^{p, 1}\left(\mathbf{R}^{2 d}\right) \# M^{q, 1}\left(\mathbf{R}^{2 d}\right) \subseteq M^{r, 1}\left(\mathbf{R}^{2 d}\right), \quad 1 / p+1 / q \geq 1 / r, \quad p, q, r \in[1, \infty],
$$

was proved. Further results on the algebraic properties of the modulation spaces considered as symbol classes were obtained by Labate in [12] from which it follows that

$$
M^{p}\left(\mathbf{R}^{2 d}\right) \# M^{p}\left(\mathbf{R}^{2 d}\right) \subseteq M^{p}\left(\mathbf{R}^{2 d}\right), \quad M^{p^{\prime}}\left(\mathbf{R}^{2 d}\right) \# M^{p}\left(\mathbf{R}^{2 d}\right) \subseteq M^{p^{\prime}}\left(\mathbf{R}^{2 d}\right), \quad p \in[1,2] .
$$

Here we set $M^{p}=M^{p, p}$, and $p^{\prime} \in[1, \infty]$ denotes the conjugate exponent of $p \in[1, \infty]$, i. e. $1 / p+1 / p^{\prime}=1$. We note that if $p=2$, then it follows that $M^{2}$ is an algebra under the Weyl product. Since $M^{2}=L^{2}$, it therefore follows that $L^{2}$ also is an algebra under this product, which was at first proved by Pool in [13.

In Section 3 we prove a general result, extending all these results (cf. Theorem 3.1 , which agrees with Theorem $0.3^{\prime}$ in [10] after general (weighted) modulation spaces in the latter result is replaced by classical (non-weighted) modulation spaces). We also remark that the results in [10] comprise and generalize all results in this context that we are aware of, when the weights involved are bounded by certain polynomials. Furthermore it seems that the arguments in [10] also work in more general cases, e.g. for weights moderated by subexponential, or sometimes also by exponential functions.

2. Modulation spaces. In this section we recall some basic facts about classical modulation spaces and pseudo-differential operators.

We start by discussing some properties of the symplectic Fourier transform and related objects. The even-dimensional vector space $\mathbf{R}^{2 d}$ is a (real) symplectic vector space with the (standard) symplectic form

$$
\sigma(X, Y)=\sigma((x, \xi) ;(y, \eta))=\langle y, \xi\rangle-\langle x, \eta\rangle
$$

where $\langle\cdot, \cdot\rangle$ denotes the usual scalar product on $\mathbf{R}^{d}$.

The symplectic Fourier transform for $a \in \mathscr{S}\left(\mathbf{R}^{2 d}\right)$ is defined by the formula

$$
\left(\mathscr{F}_{\sigma} a\right)(X)=\widehat{a}(X)=\pi^{-d} \int a(Y) e^{2 i \sigma(X, Y)} d Y .
$$

Then $\mathscr{F}_{\sigma}^{-1}=\mathscr{F}_{\sigma}$ is continuous on $\mathscr{S}\left(\mathbf{R}^{2 d}\right)$, and extends as usual to a homeomorphism on $\mathscr{S}^{\prime}\left(\mathbf{R}^{2 d}\right)$, and to a unitary map on $L^{2}\left(\mathbf{R}^{2 d}\right)$. The (symplectic) short-time Fourier transform (STFT) of $a \in \mathscr{S}^{\prime}\left(\mathbf{R}^{2 d}\right)$ with respect to a window function $\chi \in \mathscr{S}\left(\mathbf{R}^{2 d}\right)$ is defined by

$$
V_{\chi} a(X, Y)=\mathscr{F}_{\sigma}\left(a \tau_{X} \chi\right)(Y), X, Y \in \mathbf{R}^{2 d} .
$$

Here and in what follows $\tau_{X}$ is the translation operator, defined by $\tau_{X} f(Y)=f(Y-X)$. Then $V_{\chi} a$ is smooth and polynomially bounded (cf. [6]). The STFT of $a \in \mathscr{S}^{\prime}\left(\mathbf{R}^{2 d}\right)$ enjoys the orthogonality relation

$$
\left(V_{\chi_{1}} a, V_{\chi_{2}} \varphi\right)=(a, \varphi)\left(\chi_{1}, \chi_{2}\right), \quad \varphi, \chi_{1}, \chi_{2} \in \mathscr{S}\left(\mathbf{R}^{2 d}\right),
$$

where $(\cdot, \cdot)=(\cdot, \cdot)_{L^{2}\left(\mathbf{R}^{2 d}\right)}$ denotes the extension of the $L^{2}$-product on $C_{0}^{\infty}\left(\mathbf{R}^{2 d}\right)$ to a product between appropriate function and distribution spaces, and their duals (cf. [5]). We also set $\langle a, \varphi\rangle=(a, \bar{\varphi})$ for appropriate distributions $a$ and $\varphi$ on $\mathbf{R}^{2 d}$. 
Next assume that $p, q \in[1, \infty]$ and that $\chi \in \mathscr{S}\left(\mathbf{R}^{2 d}\right) \backslash\{0\}$ is fixed (but arbitrary). Then the classical modulation space $M^{p, q}\left(\mathbf{R}^{2 d}\right)$ is defined as the set of $a \in \mathscr{S}^{\prime}\left(\mathbf{R}^{2 d}\right)$ such that

$$
\|a\|_{M^{p, q}}=\left(\int\left(\int\left|V_{\chi} a(X, Y)\right|^{p} d X\right)^{q / p} d Y\right)^{1 / q}
$$

is finite. Here the $L^{p}$-norm or $L^{q}$-norm should be replaced by the $L^{\infty}$-norm when $p=\infty$ or $q=\infty$. For simplicity we set $M^{p, p}=M^{p}$, and let $\mathscr{M}^{p, q}\left(\mathbf{R}^{2 d}\right)$ be the completion of $\mathscr{S}\left(\mathbf{R}^{2 d}\right)$ in the norm $\|\cdot\|_{M^{p, q}}$.

In the following proposition we collect some properties of modulation spaces that are important in this paper. We omit the proof, since it can be found in [1, 2, 6, 16].

Proposition 2.1. Assume that $p, q \in[1, \infty]$. Then the following statements are true:

(i) the space $M^{p, q}\left(\mathbf{R}^{2 d}\right)$ is a Banach space which is independent of $\chi \in \mathscr{S}\left(\mathbf{R}^{2 d}\right) \backslash\{0\}$, and different $\chi$ give rise to equivalent norms;

(ii) if $p_{1}, p_{2}, q_{1}, q_{2} \in[1, \infty]$ and $p_{1} \leq p_{2}, q_{1} \leq q_{2}$ then

$$
\mathscr{S}\left(\mathbf{R}^{2 d}\right) \subseteq M^{p_{1}, q_{1}}\left(\mathbf{R}^{2 d}\right) \subseteq M^{p_{2}, q_{2}}\left(\mathbf{R}^{2 d}\right) \subseteq \mathscr{S}^{\prime}\left(\mathbf{R}^{2 d}\right) ;
$$

(iii) the $L^{2}$-product $(\cdot, \cdot)$ on $C_{0}^{\infty}\left(\mathbf{R}^{2 d}\right)$ extends to a continuous sesquilinear form on $M^{p, q}\left(\mathbf{R}^{2 d}\right) \times M^{p^{\prime}, q^{\prime}}\left(\mathbf{R}^{2 d}\right)$. Furthermore, $\|a\|=\sup |(a, b)|$, with supremum taken over all $b \in C_{0}^{\infty}\left(\mathbf{R}^{2 d}\right)$ such that $\|b\|_{M^{p^{\prime}, q^{\prime}}} \leq 1$, is a norm equivalent to $\|a\|_{M^{p, q}}$. If $p, q<\infty$, then the dual space of $M^{p, q}$ can be identified with $M^{p^{\prime}, q^{\prime}}$ through the form $(\cdot, \cdot)$;

(iv) $\mathscr{M}^{p, q} \subseteq M^{p, q}$ with equality if and only if $p<\infty, q<\infty$. Furthermore, $\mathscr{S}\left(\mathbf{R}^{2 d}\right)$ is weakly dense in $M^{p, q}\left(\mathbf{R}^{2 d}\right)$ provided $(p, q) \neq(1, \infty)$ and $(p, q) \neq(\infty, 1)$.

The next result concerns (complex) interpolation and modulation spaces. Again we omit the proof, since it can be found in [4].

Proposition 2.2. Assume that $0 \leq \theta \leq 1$ and $p, q, p_{1}, p_{2}, q_{1}, q_{2} \in[1, \infty]$ are such that

Then $\left(\mathscr{M}^{p_{1}, q_{1}}, \mathscr{M}^{p_{2}, q_{2}}\right)_{[\theta]}=\mathscr{M}^{p, q}$.

$$
\frac{1}{p}=\frac{1-\theta}{p_{1}}+\frac{\theta}{p_{2}}, \quad \frac{1}{q}=\frac{1-\theta}{q_{1}}+\frac{\theta}{q_{2}} .
$$

Next we recall some results for pseudo-differential operators with symbols in modulation spaces. If $p_{j}, q_{j} \in[1, \infty], j=0,1,2$, are such that

$$
\frac{1}{p_{1}}-\frac{1}{p_{2}}=\frac{1}{q_{1}}-\frac{1}{q_{2}}=1-\frac{1}{p_{0}}-\frac{1}{q_{0}}, \quad q_{0} \leq p_{2}, q_{2} \leq p_{0},
$$

and $a \in M^{p_{0}, q_{0}}\left(\mathbf{R}^{2 d}\right)$, then it is proved in [8, 16] that $a^{w}(x, D)$ extends uniquely to a continuous operator from $M^{p_{1}, q_{1}}\left(\mathbf{R}^{d}\right)$ to $M^{p_{2}, q_{2}}\left(\mathbf{R}^{d}\right)$. Some extensions of this result which involves weighted modulation spaces can be found in 17, 19. Moreover, each $a$ in $M^{p, q}\left(\mathbf{R}^{2 d}\right)$ gives rise to $L^{2}$-bounded Weyl operators if and only if $p \in[1, \infty]$ and $1 \leq$ $q \leq \min \left(2, p^{\prime}\right)$. (Cf. 9].) Some Schatten-von Neumann properties for pseudo-differential operators with symbols in modulation spaces can be found in [7, 16, 19].

3. Continuity of the Weyl product on modulation spaces. In this section we discuss algebraic properties of the Weyl product acting on modulation spaces, and prove 
a general result on continuity of the Weyl product when acting on classical modulation spaces (cf. Theorem 3.1 below).

The following result agrees with Theorem $0.3^{\prime}$ in [10, if all weight functions in the latter theorem are trivially equal to 1.

TheOREM 3.1. Assume that $p_{j}, q_{j} \in[1, \infty], j=0,1,2$, satisfy

$$
\begin{aligned}
& \frac{1}{p_{1}}+\frac{1}{p_{2}}+\frac{1}{q_{1}}+\frac{1}{q_{2}}=1+\frac{1}{p_{0}}+\frac{1}{q_{0}}, \quad q_{1}, q_{2} \leq q_{0}, \\
& 0 \leq \frac{1}{p_{1}}+\frac{1}{p_{2}}-\frac{1}{p_{0}} \leq \frac{1}{p_{j}}, \frac{1}{q_{j}} \leq \frac{1}{q_{1}}+\frac{1}{q_{2}}-\frac{1}{q_{0}}, \quad j=0,1,2 .
\end{aligned}
$$

Then the map (2) extends uniquely to a continuous map from $M^{p_{1}, q_{1}}\left(\mathbf{R}^{2 d}\right) \times M^{p_{2}, q_{2}}\left(\mathbf{R}^{2 d}\right)$ to $M^{p_{0}, q_{0}}\left(\mathbf{R}^{2 d}\right)$, and for some constant $C>0$, the bound 3 holds for every a $\in$ $M^{p_{1}, q_{1}}\left(\mathbf{R}^{2 d}\right)$ and $b \in M^{p_{2}, q_{2}}\left(\mathbf{R}^{2 d}\right)$.

For the proof we recall that $M^{2}=L^{2}$ is an algebra under the Weyl product, i. e. $\|a \# b\|_{L^{2}} \leq C\|a\|_{L^{2}}\|b\|_{L^{2}}$. (See e.g. [5].)

Proof. By Theorem 4.1 in [15], the result follows for $q_{j}=1$, and by duality the result also follows for $1 / p_{1}+1 / p_{2}=1 / p_{0}$, and $q_{0}=q_{1}=q_{2}^{\prime}=\infty$ or $q_{0}=q_{1}^{\prime}=q_{2}=\infty$. By Proposition 2.2 and interpolation, the result holds for $q_{1}=1$ and $q_{2}=q_{0}$, or $q_{2}=1$ and $q_{1}=q_{0}$. Furthermore, the result holds for $p_{q}=q_{j}=2$, since the Weyl product is continuous on $L^{2}=M^{2}$. The result now follows for general $p_{j}$ and $q_{j}$ by interpolating these results, using Proposition 2.2. (See Theorem $0.3^{\prime}$ and its proof in 10 for further details.)

We finish the section by listing some immediate consequences of Theorem 3.1 .

Corollary 3.2. Assume that $p \in[1, \infty]$ and $1 \leq q \leq \min \left(p, p^{\prime}\right)$. Then the Weyl product extends to a continuous multiplication from $M^{p, q} \times M^{p, q}$ to $M^{p, q}$, and there exists $C>0$ such that

$$
\|a \# b\|_{M^{p, q}} \leq C\|a\|_{M^{p, q}}\|b\|_{M^{p, q}},
$$

holds for all $a \in M^{p, q}$ and $b \in M^{p, q}$, i. e. $M^{p, q}$ is an algebra under the Weyl product.

We note that the condition $1 \leq q \leq \min \left(p, p^{\prime}\right)$ in Corollary 3.2 is also necessary in order for $M^{p, q}$ to be an algebra under the Weyl product (cf. [10, Theorem 3.6]).

REMARK 3.3. Assume that $p, q, r, s \in[1, \infty]$ are such that $s \leq 2, r \leq s^{\prime}$ and $(1 / p, 1 / q)$ belongs to the square with corners at

$$
\left(1 / s^{\prime}, 1 / s^{\prime}\right), \quad\left(1 / s^{\prime}, 1 / s\right), \quad\left(1 / s, 1 / s^{\prime}\right), \quad \text { and } \quad(1 / s, 1 / s) .
$$

By Theorem 3.1 it follows that

$$
M^{p, q} \# M^{r, s} \subseteq M^{p, q}, \quad M^{r, s} \# M^{p, q} \subseteq M^{p, q} .
$$

(Cf. Remark 2.17 in [10].) If in addition $s \leq r$, then it follows in particular that $M^{r, s}$ is a Banach algebra and $M^{p, q}$ is an $M^{r, s}$-module under the Weyl product.

On the other hand, in Section 3 in [10] it is proved that the module properties in Remark 3.3 are sharp in the sense that (7) fails when $s^{\prime}<r$, and that $M^{r, s}$ is not an algebra when $r<s$. 
Acknowledgments. The second author (Joachim Toft) is grateful to Prof. Yoshino, Prof. Sugimoto and Prof. Furutani for the nice hospitality during his stay in Japan in November 2007.

\section{References}

[1] H. G. Feichtinger, Banach convolution algebras of Wiener's type, in: Functions, Series, Operators in Budapest, Colloquia Math. Soc. J. Bolyai, North-Holland, Amsterdam, 1980.

[2] H. G. Feichtinger, Modulation spaces on locally compact abelian groups, Technical report, University of Vienna, Vienna, 1983; also in: M. Krishna et al. (eds.), Wavelets and Their Applications, Allied Publ., New Delhi, 2003, 99-140.

[3] H. G. Feichtinger, Modulation spaces: looking back and ahead, Sampl. Theory Signal Image Process. 5 (2006), 109-140.

[4] H. G. Feichtinger and K. H. Gröchenig, Banach spaces related to integrable group representations and their atomic decompositions, II, Monatsh. Math. 108 (1989), 129-148.

[5] G. B. Folland, Harmonic Analysis in Phase Space, Princeton U. P., Princeton, 1989.

[6] K. H. Gröchenig, Foundations of Time-Frequency Analysis, Birkhäuser, Boston, 2001.

[7] K. H. Gröchenig and C. Heil, Modulation spaces and pseudo-differential operators, Integral Equations Operator Theory 34 (1999), 439-457.

[8] K. H. Gröchenig and C. Heil, Modulation spaces as symbol classes for pseudodifferential operators, in: M. Krishna et al. (eds.), Wavelets and Their Applications, Allied Publ., New Delhi, 2003, 151-170.

[9] K. H. Gröchenig and C. Heil, Counterexamples for boundedness of pseudodifferential operators, Osaka J. Math. 41 (2004), 681-691.

[10] A. Holst, J. Toft and P. Wahlberg, Weyl product algebras and modulation spaces, J. Funct. Anal. 251 (2007), 463-491.

[11] L. Hörmander, The Analysis of Linear Partial Differential Operators, Vols. I, III, Springer, Berlin, 1983, 1985.

[12] D. Labate, Time-frequency analysis of pseudodifferential operators, Monatsh. Math. 133 (2001), 143-156.

[13] J. C. T. Pool, Mathematical aspects of the Weyl correspondence, J. Math. Phys. 7 (1966), $66-76$.

[14] J. Sjöstrand, An algebra of pseudodifferential operators, Math. Res. L. 1 (1994), 185-192.

[15] J. Toft, Subalgebras to a Wiener type algebra of pseudo-differential operators, Ann. Inst. Fourier 51 (2001), 1347-1383.

[16] J. Toft, Continuity properties for modulation spaces with applications to pseudo-differential calculus, I, J. Funct. Anal. 207 (2004), 399-429.

[17] J. Toft, Continuity properties for modulation spaces with applications to pseudo-differential calculus, II, Ann. Global Anal. Geom. 26 (2004), 73-106.

[18] J. Toft, Convolution and embeddings for weighted modulation spaces, in: P. Boggiatto et al. (eds.), Advances in Pseudo-Differential Operators, Operator Theory: Advances and Applications 155, Birkhäuser Verlag, Basel, 2004, 165-186.

[19] J. Toft, Continuity and Schatten properties for pseudo-differential operators on modulation spaces in: J. Toft et al. (eds.), Modern Trends in Pseudo-Differential Operators, Operator Theory: Advances and Applications 172, Birkhäuser Verlag, Basel, 2007, 173-206. 